\title{
Histone deacetylase 5 promotes the proliferation of glioma cells by upregulation of Notch 1
}

\author{
QUAN LIU $^{1 *}$, JIE-MIN ZHENG $^{1 *}$, JIA-KANG CHEN $^{1}$, XIAN-LEI YAN ${ }^{1}$, \\ HONG-MOU CHEN ${ }^{1}$, WEI-XIA NONG ${ }^{2}$ and HE-QING HUANG ${ }^{1}$ \\ ${ }^{1}$ Department of Neurosurgery, The Fourth Affiliated Hospital of Guangxi Medical University, Liuzhou, Guangxi 545005; \\ ${ }^{2}$ Department of Histology and Embryology, School of Basic Medicine, Guangxi Medical University, \\ Nanning, Guangxi 530021, P.R. China
}

Received November 17, 2013; Accepted May 14, 2014

DOI: $10.3892 / \mathrm{mmr} .2014 .2395$

\begin{abstract}
Histone deacetylases (HDACs) constitute a family of enzymes that play important roles in the epigenetic regulation of gene expression and contribute to the growth, differentiation and apoptosis of cancer cells. However, the biological function of HDAC 5 in glioma cells has not been fully understood. In the present study, we found that the mRNA and protein levels of HDAC5 are increased in human glioma tissues and cell lines. In addition, overexpression of HDAC5 promoted proliferation of glioma cells, as measured by the MTT assay. By contrast, HDAC5 gene silencing using small interfering RNA (siRNA) inhibited cell proliferation. Furthermore, we demonstrated that HDAC5 enhances Notch 1 expression at both the mRNA and the protein level in glioma cell lines. Taken together, these results demonstrated, for the first time to the best of our knowledge, that HDAC5 promotes glioma cell proliferation, and suggest that this effect involves the upregulation of Notch 1. Therefore, our study may provide a novel therapeutic target for treatment of gliomas.
\end{abstract}

\section{Introduction}

Gliomas are the most common primary tumor type of the central nervous system and have a poor prognosis (1). In a meta-analysis of 12 randomized clinical trials, the overall 1-year survival rate of high-grade gliomas, such as glioblastomas and anaplastic astrocytomas, was $40 \%$, and only slightly higher $(46 \%)$ following combined radiotherapy and chemotherapy (2). Current clinical treatment of glioma includes radiotherapy, chemotherapy and surgical operation (3). However, an increasing number of studies have shown that the

Correspondence to: Dr He-Qing Huang, Department of Neurosurgery, The Fourth Affiliated Hospital of Guangxi Medical University, 1 Liushi Road, Liuzhou, Guangxi 545005, P.R. China

E-mail: huangheqing2013@126.com

*Contributed equally

Key words: glioma, cell proliferation, HDAC5, Notch 1 resistance of glioma cells to conventional drugs is becoming a challenging issue. Therefore, it is urgent to develop new and effective therapeutic methods for the treatment of gliomas (4).

The histone deacetylase (HDAC) family contains a total of 18 proteins, grouped into classes I-IV based on their homology and structure. Classes I, II and IV contain 11 family members, which are called classical HDACs, whereas the 7 members of the class III are referred to as sirtuins $(5,6)$. HDAC5 belongs to the class II, and has been shown to constitute an important regulator of cell proliferation, cell-cycle progression and apoptosis $(7,8)$. Additional members of the HDAC family were demonstrated to play critical roles in tumor initiation, proliferation and metastasis, such as SIRT6 (9). A number of HDAC inhibitors possess the ability to promote apoptosis and inhibit proliferation in tumor cells and animal models (10). In addition, a clinical study previously highlighted the potential of HDAC inhibitors to be used as anticancer agents (11). However, the biological function of HDAC5 in human glioma has never been investigated to date. Therefore, in the present study, we examined the expression of HDAC5 in human glioma samples and further investigated its biological function.

\section{Materials and methods}

Tissue samples and ethics. Glioma and distant healthy tissues were collected from therapeutic surgery at the Department of Neurosurgery, the Fourth Affiliated Hospital of Guangxi Medical University (Guangxi, China). All samples were obtained with informed consent from the participants, and this study was approved by the Institutional Review Board of the Fourth Affiliated Hospital of Guangxi Medical University.

Cell culture. The human glioma cell lines U87 and LN-229 were obtained from the Shanghai Institute of Cell Biology, Chinese Academy of Sciences (Shanghai, China). Cells were cultured in Dulbecco's modified Eagle's medium supplemented with $10 \%$ fetal bovine serum, streptomycin $(100 \mathrm{mg} / \mathrm{ml})$ and penicillin $(100 \mathrm{U} / \mathrm{ml})$. Cultured cells were maintained at $37^{\circ} \mathrm{C}$ and $5 \% \mathrm{CO}_{2}$ in a humid environment, and were passaged when the confluency had reached $80 \%$. 
A

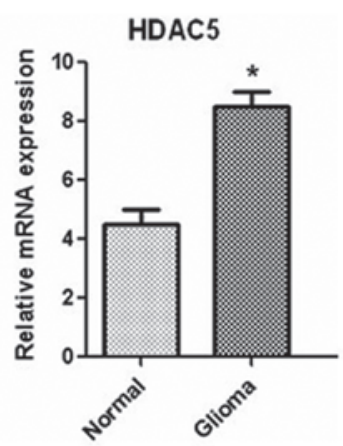

$\mathbf{C}$

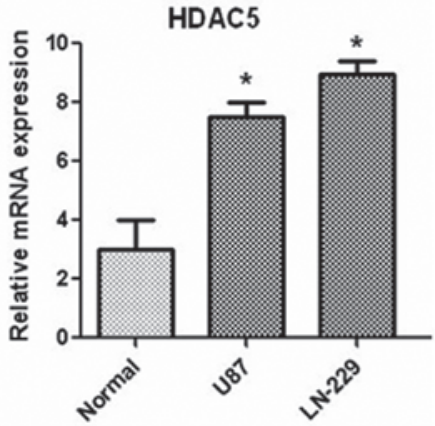

B

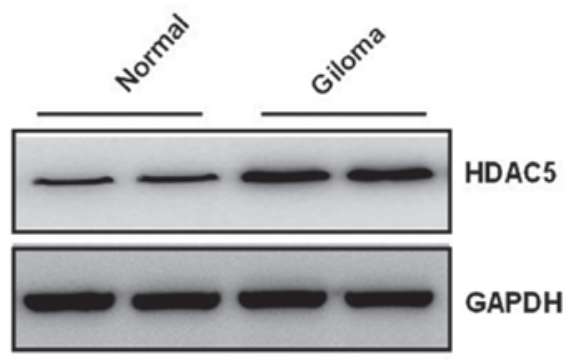

D

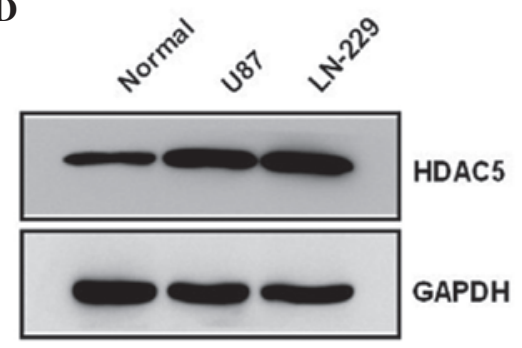

Figure 1. Upregulation of histone deacetylase 5 (HDAC5) in human glioma tissues and cell lines. The (A) mRNA and (B) protein levels of HDAC5 were determined by reverse transcription-quantitative polymerase chain reaction (RT-qPCR) and western blotting in human glioma and healthy tissues. (C) RT-qPCR and (D) western blotting were performed to determine the expression of HDAC5 in healthy human astrocytes and the glioma cell lines U87 and LN-229. ${ }^{*} \mathrm{P}<0.05$ compared to healthy tissues/astrocytes (normal). GAPDH, glyceraldehyde 3-phosphate dehydrogenase.

Healthy human astrocytes were cultured in astrocyte medium (Dulbecco's modified Eagle's medium/F12 supplemented with $10 \%$ fetal bovine serum, Gibco Laboratories, Grand Island, NY).

Plasmid construction, small interfering RNA (siRNA) and transfection. The complementary DNA (cDNA) fragment encoding HDAC5 was obtained by reverse transcription (RT) performed on total RNA extracted from the glioma cell line U87, using the Takara RNA PCR kit reagents (Takara Bio Inc., Tokyo, Japan). RNA extraction was performed using the TRIzol reagent (Invitrogen Life Technologies, Carlsbad, CA, USA) following the manufacturer's instructions. The cDNA was then amplified by polymerase chain reaction (PCR) using the following primers: forward, 5'-GGA ATT CAT GAA GTT GGA GGT GTT CGT C-3', and reverse, 5'-CCT CGA GCG CTA CTC AGG CTA GGA GCG TCT CCA C-3'.

The PCR product was cloned into the mammalian expression vector pcDNA3.1 (+)-flag (Invitrogen Life Technologies). Two independent oligos targeting HDAC5 (siRNA-HDAC5), siRNA Notch 1 and scrambled control siRNA were purchased from Invitrogen Life Technologies. Cells were transfected with lipofectamine 2000 (Invitrogen Life Technologies) according to the manufacturer's instructions.

Quantitative (q)PCR. In order to quantify the transcripts of the genes of interest, qPCR was performed using the SYBR ${ }^{\circledR}$ Premix Ex Taq ${ }^{\mathrm{TM}}$ kit (Takara Bio Inc.) on an Applied Biosystems ${ }^{\circledR}$ ABI 7500 system (Thermo Fisher Scientific). The cycling parameters were as follows: an initial stage of $95^{\circ} \mathrm{C}$ for $30 \mathrm{sec}$, followed by a two step program of $95^{\circ} \mathrm{C}$ for $5 \mathrm{sec}$ and $60^{\circ} \mathrm{C}$ for $31 \mathrm{sec}$ over 40 cycles, performed in triplicate. The qPCR data were analyzed using the $2^{-\Delta \Delta C t}$ method (12).

MTT assay. Proliferation of cells was determined using an MTT assay (Sigma, St. Louis, MO, USA). Briefly, the glioma cells transfected with indicated oligonucleotides were seeded into 96-well plates at a density of $3 \times 10^{4}$ (cells/well). Subsequently, $10 \mathrm{ml}$ of $5 \mathrm{mg} / \mathrm{ml}$ MTT was added and incubated in the dark at $37^{\circ} \mathrm{C}$ for $2 \mathrm{~h}$. The absorbance was determined at a wavelength of $490 \mathrm{~nm}$ using a BioRad microplate reader (FluoDia T70; Photon Technology International, Inc., Lawrenceville, NJ, USA).

Western blotting. Cells were harvested by trypsinization and lysed in buffer (Beyotime Institute of Biotechnology, Shanghai, China). The proteins were then subjected to sodium dodecyl sulfate-polyacrylamide gel electrophoresis and transferred onto a polyvinylidene fluoride membrane (Millipore, Billerica, MA, USA). The membranes were blocked in phosphate-buffered saline (PBS) $/ 0.1 \%$ Tween-20 with 5\% nonfat dry milk and then incubated with primary antibodies in PBS/0.1\% Tween-20 with 0.1-5\% nonfat dry milk. Antibodies directed against HDAC5 (rabbit polyclonal, dilution 1:2,000), Notch 1 (rabbit polyclonal, dilution 1:2,000) and glyceraldehyde 3-phosphate dehydrogenase (GAPDH; mouse monoclonal, dilution 1:5,000) were purchased from Santa Cruz Biotechnology, Inc. (Santa Cruz, CA, USA) with GAPDH used as a loading control.

Statistical analysis. All data were expressed as mean \pm standard error of the mean, and were analyzed using the SPSS software (IBM, Armonk, NY, USA). P-values (P) for the comparisons between groups were determined by analysis of variance 


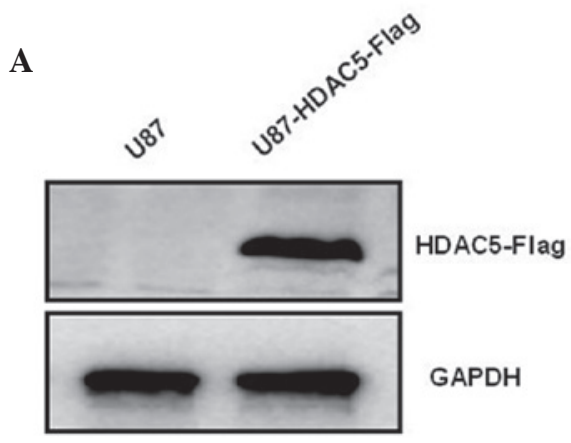

B

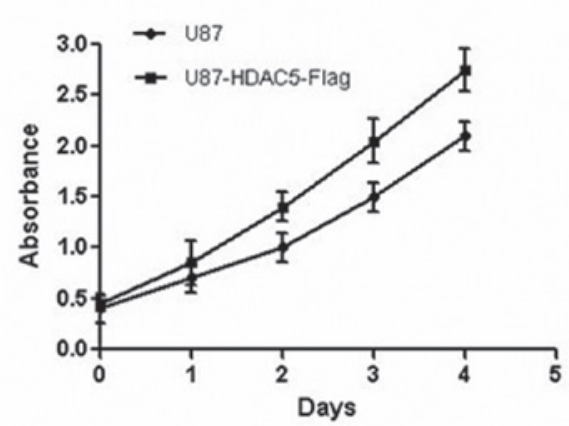

Figure 2. Histone deacetylase 5 (HDAC5) overexpression promotes the proliferation of glioma cells. (A) HDAC5 expression was determined by western blotting in U87 glioma cells transfected with a plasmid bearing the HDAC5 gene with a flag tag. (B) The proliferation of U87 glioma cells following transfection with the plasmid bearing the HDAC5-flag tag was measured using the MTT assay. GAPDH, glyceraldehyde 3-phosphate dehydrogenase.

A

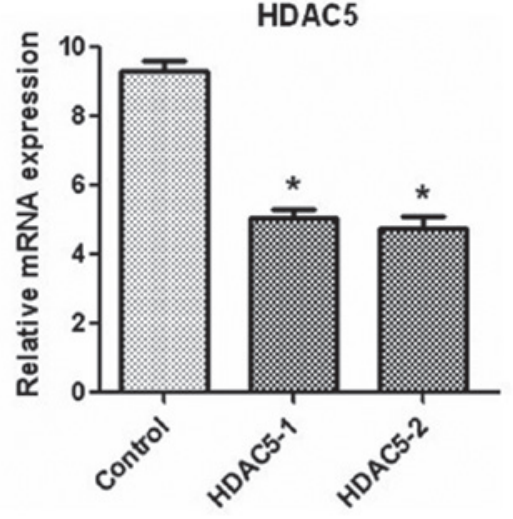

B

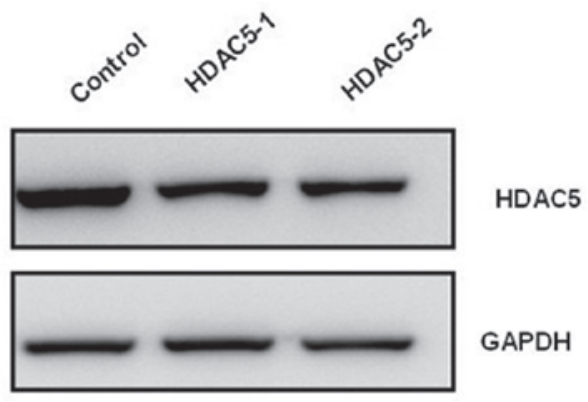

C

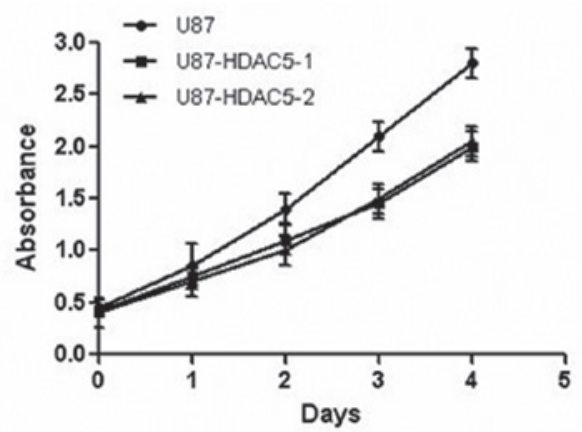

Figure 3. Downregulation of the histone deacetylase 5 (HDAC5) gene inhibits glioma cell proliferation. (A) Reverse transcription-quantitative polymerase chain reaction (RT-qPCR) and (B) western blotting were used to measure the HDAC5 expression at the mRNA and protein level in U87 glioma cells transfected with small interfering RNA (siRNA) oligos targeting the HDAC5 gene (HDAC5-1 and -2 cell lines). * $<0.05$ compared to the control (cells transfected with the scramble siRNA). (C) Cell proliferation was measured using the MTT assay in U87 cells and U87 cells transfected with HDAC5 siRNA oligos. GAPDH, glyceraldehyde 3-phosphate dehydrogenase.

(ANOVA), with $\mathrm{P}<0.05$ considered to indicate statistically significant differences.

\section{Results}

HDAC5 expression is increased in human glioma tissues and cells. We first analyzed the expression of the HDAC5 gene in 20 paired glioma and adjacent non-tumor healthy tissues by
RT-qPCR. Results showed that the mRNA level of HDAC5 is significantly increased in human cancer compared to healthy tissues (Fig. 1A). Then, western blotting was used to determine the protein expression of HDAC5. We found that the protein level of HDAC5 is also increased in glioma samples (Fig. 1B). Furthermore, two glioma cell lines were analyzed by RT-qPCR and western blotting. As shown in Fig. 1C and D, higher expression of HDAC5, at both the mRNA and the protein level, 
A

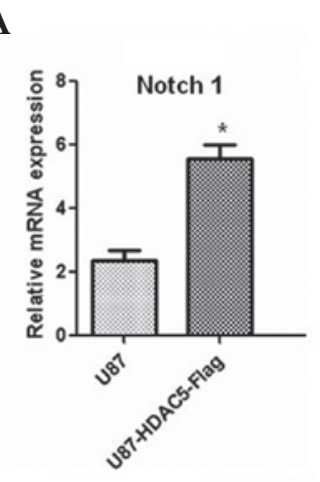

D

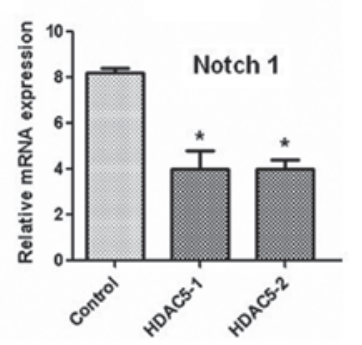

B

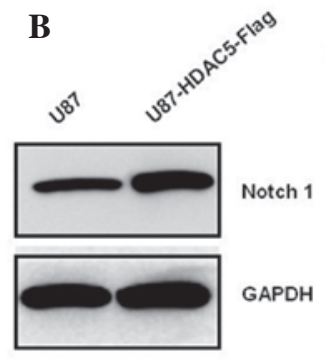

C

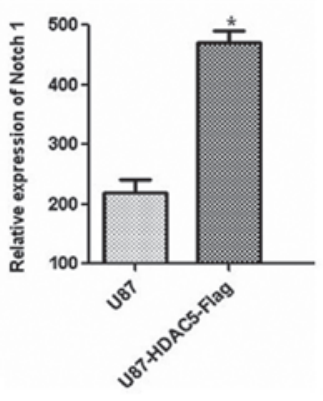

$\mathbf{F}$

$\mathbf{E}$

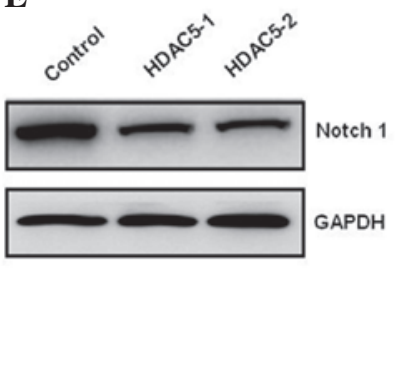

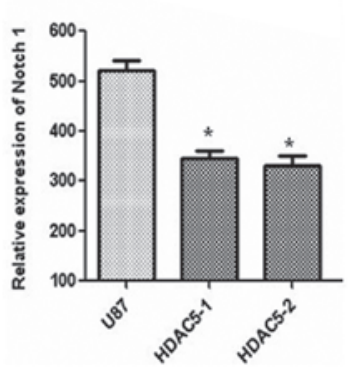

Figure 4. Histone deacetylase 5 (HDAC5) positively regulates Notch 1 expression. (A) The mRNA and (B and C) the protein level of Notch 1 in U87 cells overexpressing HDAC5. The mRNA level was determined by reverse transcription-quantitative polymerase chain reaction (RT-qPCR), and the protein level was measured by western blotting. Relative band intensities of each protein were quantified by densitometry. ${ }^{*} \mathrm{P}<0.05$ compared to U87 cells with normal expression of HDAC5. (D) The mRNA level and (E and F) the protein level of Notch 1 in HDAC5-silenced U87 cells, measured by RT-qPCR and western blotting. " $\mathrm{P}<0.05$ compared to the control (cells transfected with the scramble small interfering RNA instead of the one targeting HDAC5. GAPDH, glyceraldehyde 3-phosphate dehydrogenase.

A

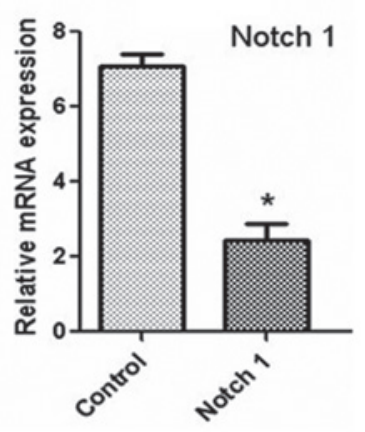

B

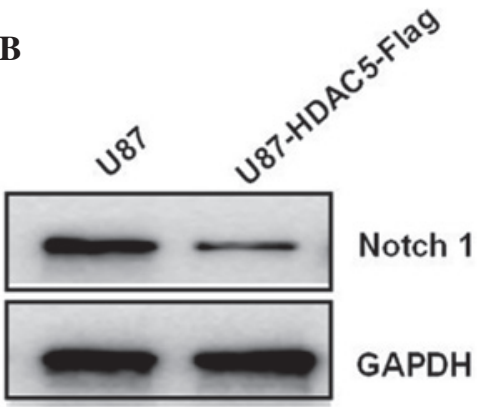

C

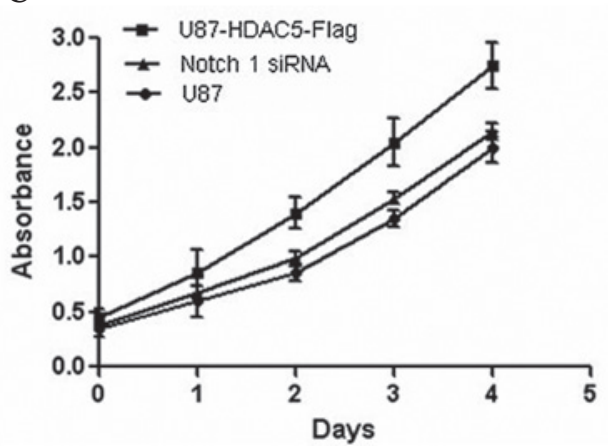

Figure 5. Histone deacetylase 5 (HDAC5) promotes glioma cell proliferation by upregulating Notch 1. (A and B) Reverse transcription-quantitative polymerase chain reaction and western blotting were performed to detect the mRNA and protein levels of Notch 1 in U87 cells transfected with small interfering (siRNA) oligos targeting the Notch 1 gene. ${ }^{*} \mathrm{P}<0.05$ compared to the control (cells transfected with the scramble small interfering RNA). (C) The MTT assay was used to measure the proliferation of U87 cells overexpressing HDAC5 with or without Notch 1 silencing. GAPDH, glyceraldehyde 3-phosphate dehydrogenase. 
was observed in the two lines compared to healthy human astrocytes, suggesting that HDAC5 expression is upregulated in glioma tissues and cell lines.

HDAC5 overexpression promotes proliferation of glioma cells. To further investigate the biological role of HDAC5 in human glioma, U87 cells were transfected with a plasmid containing the HDAC5 gene, which allowed its overexpression (Fig. 2A). Results of the MTT assay further showed that HDAC5 overexpression promotes glioma cell proliferation (Fig. 2B). In addition, U87 cells were transfected with siRNA targeting HDAC5. Efficient silencing of HDAC5 was achieved with two independent siRNA oligos compared to scramble siRNA-transfected U87 cells (Fig. 3A and B). Silencing of HDAC5 in these cells inhibited cell proliferation (Fig. 3C). Similar results were also observed in LN-229 cells (data not shown). Taken together, our results demonstrated that HDAC5 promotes the proliferation of glioma cells.

HDAC5 promotes glioma cell proliferation via Notch 1 upregulation. Next, we investigated the molecular mechanism underlying the effect of HDAC5 on cell proliferation. The results demonstrated that the Notch 1 mRNA level was significantly increased in the cells overexpressing HDAC5 (Fig. 4A). An increase in the Notch 1 protein level was also observed by western blotting (Fig. 4B and C). Consistent with these results, silencing of the HDAC5 gene markedly reduced Notch 1 expression at the mRNA (Fig. 4D) and the protein level (Fig. 4E and F). These results indicated that HDAC5 may act as an upstream regulator of Notch 1. Furthermore, the Notch 1 gene was silenced using siRNA oligos, and the protein levels of Notch 1 were also reduced (Fig. 5A and B). The decreased expression of Notch 1 attenuated the effect of HDAC5 on glioma cell proliferation (Fig. 5C). Taken together, these results indicated that HDAC5 promotes cell proliferation by upregulating Notch 1 .

\section{Discussion}

It has been reported that certain members of the HDAC family play critical roles in promoting carcinogenesis (9). However, the biological function of HDAC5 in glioma cells remains poorly understood. In the current study, the biological function of HDAC5 was investigated in glioma tissues and cell lines for the first time, to the best of our knowledge.

HDACs remove the acetyl groups from the $\mathrm{N}$-acetylly-sines on a histone and modify the chromatin structure, therefore modulating the expression levels of numerous genes. Their aberrant expression is closely related to tumor initiation and development (13). In our study, we found that both the mRNA and protein expression of HDAC5 were significantly increased in glioma tissues and cell lines.

The class II HDAC family member HDAC5 has been shown to be an important regulator of cell-cycle progression, proliferation and apoptosis in numerous cancer cell lines and animal models (14). For example, HDAC5 was shown to translocate from the nucleus to the cytoplasm during myoblast differentiation and suppress the expression of the cell-cycle activator cyclin D3, confirming its involvement in cell differentiation and proliferation $(15,16)$. A recent study demonstrated that HDAC5 and HDAC9 are significantly upregulated in high-risk medulloblastoma in comparison to low-risk medulloblastoma samples, and that their expression is associated with poor survival, indicating that HDAC5 and HDAC9 may be valuable markers for risk stratification (17). Another study reported that HDAC5 upregulates Twist 1, and revealed a so-far unknown link between HDAC5 and osteosarcoma progression (18).

In our study, overexpression of HDAC5 enhanced glioma cell proliferation. In addition, HDAC5 silencing using siRNA inhibited glioma cell proliferation, indicating that HDAC5 may be a positive regulator of glioma cell proliferation.

The Notch family of proteins plays an important role in cell proliferation, differentiation and apoptosis. The Notch 1 protein shares structural features with other members of this family, such as the presence of an extracellular domain consisting of multiple epidermal growth factor-like repeats and an intracellular domain consisting of multiple, different domain types (19). A number of studies have shown that Notch 1 is overexpressed in cancer cell lines, including gliomas (20). Downregulation of Notch 1 and its ligands by RNA interference induces apoptosis and inhibits proliferation in multiple glioma cell lines. Therefore, Notch 1 has been proposed to constitute a promising therapeutic target in the treatment of glioma $(21,22)$. Our study found that HDAC5 significantly increases the expression of Notch 1. Furthermore, Notch 1 silencing attenuated the proliferative effect of HDAC5 on glioma cells.

In conclusion, our study demonstrated that HDAC5 promotes the proliferation of glioma cells via upregulation of Notch 1 and may provide novel therapeutic targets in the treatment of gliomas.

\section{References}

1. Medina Villaamil V, Alvarez García A, Aparicio Gallego G, et al: Tissue array analysis for the differentiation of gliosis from gliomas. Mol Med Rep 4: 451-457, 2011.

2. Stewart LA: Chemotherapy in adult high-grade glioma: a systematic review and meta-analysis of individual patient data from 12 randomised trials. Lancet 359: 1011-1018, 2002.

3. Wang Y, Zhou Z, Luo H, et al: Combination of tamoxifen and antisense human telomerase RNA inhibits glioma cell proliferation and anti-apoptosis via suppression of telomerase activity. Mol Med Rep 3: 935-940, 2010.

4. Lefranc F, Facchini V and Kiss R: Proautophagic drugs: a novel means to combat apoptosis-resistant cancers, with a special emphasis on glioblastomas. Oncologist 12: 1395-1403, 2007.

5. Witt O, Deubzer HE, Milde T and Oehme I: HDAC family: What are the cancer relevant targets? Cancer Lett 277: 8-21, 2009.

6. de Ruijter AJ, van Gennip AH, Caron HN, Kemp S and van Kuilenburg AB: Histone deacetylases (HDACs): characterization of the classical HDAC family. Biochem J 370: 737-749, 2003.

7. Voelter-Mahlknecht S,Ho AD and Mahlknecht U: Chromosomal organization and localization of the novel class IV human histone deacetylase 11 gene. Int J Mol Med 16: 589-598, 2005.

8. Sen N, Kumari R, Singh MI and Das S: HDAC5, a key component in temporal regulation of p53-mediated transactivation in response to genotoxic stress. Mol Cell 52: 406-420, 2013.

9. Witt O, Deubzer HE, Milde T and Oehme I: HDAC family: What are the cancer relevant targets? Cancer Lett 277: 8-21, 2009.

10. Wiegmans AP, Alsop AE, Bots M, et al: Deciphering the molecular events necessary for synergistic tumor cell apoptosis mediated by the histone deacetylase inhibitor vorinostat and the BH3 mimetic ABT-737. Cancer Res 71: 3603-3615, 2011. 
11. Munster P, Marchion D, Bicaku E, et al: Clinical and biological effects of valproic acid as a histone deacetylase inhibitor on tumor and surrogate tissues: phase I/II trial of valproic acid and epirubicin/FEC. Clin Cancer Res 15: 2488-2496, 2009.

12. Livak KJ and Schmittgen TD: Analysis of relative gene expression data using real-time quantitative PCR and the 2(-Delta Delta C(T)) Method. Methods 25: 402-408, 2001.

13. Ronsch K, Jager M, Schopflin A, Danciu M, Lassmann S and Hecht A: Class I and III HDACs and loss of active chromatin features contribute to epigenetic silencing of CDX1 and EPHB tumor suppressor genes in colorectal cancer. Epigenetics 6: 610-622, 2011

14. Khan $\mathrm{O}$ and La Thangue NB: HDAC inhibitors in cancer biology: emerging mechanisms and clinical applications. Immunol Cell Biol 90: 85-94, 2012.

15. Roy S, Shor AC, Bagui TK, Seto E and Pledger WJ: Histone deacetylase 5 represses the transcription of cyclin D3. J Cell Biochem 104: 2143-2154, 2008.

16. McKinsey TA, Zhang CL, Lu J and Olson EN: Signal-dependent nuclear export of a histone deacetylase regulates muscle differentiation. Nature 408: 106-111, 2000.
17. Milde T, Oehme I, Korshunov A, et al: HDAC5 and HDAC9 in medulloblastoma: novel markers for risk stratification and role in tumor cell growth. Clin Cancer Res 16: 3240-3252, 2010.

18. Chen J, Xia J, Yu YL, et al: HDAC5 promotes osteosarcoma progression by upregulation of Twist 1 expression. Tumour Biol 35: 1383-1387, 2013.

19. Rana NA and Haltiwanger RS: Fringe benefits: functional and structural impacts of O-glycosylation on the extracellular domain of Notch receptors. Curr Opin Struct Biol 21: 583-589, 2011.

20. Ayukawa T, Matsumoto K, Ishikawa HO, et al: Rescue of Notch signaling in cells incapable of GDP-L-fucose synthesis by gap junction transfer of GDP-L-fucose in Drosophila. Proc Natl Acad Sci USA 109: 15318-15323, 2012.

21. Hu B, Nandhu MS, Sim H, et al: Fibulin-3 promotes glioma growth and resistance through a novel paracrine regulation of Notch signaling. Cancer Res 72: 3873-3885, 2012.

22. Berry N, Gursel DB and Boockvar JA: Notch inhibition via micro-RNA blocks glioma development. Neurosurgery 70: N20-N22, 2012 